\title{
Avaliação da condição de saúde mental de estudantes de Medicina perante o cenário da pandemia da Covid-19
}

\author{
Assessing the mental health condition of Medical students in the Covid-19 pandemic scenario \\ Evaluación de la condición de salud mental de los estudiantes de Medicina en el escenario de la \\ pandemia Covid-19
}

Enzo Mugayar Campanholo ORCID: https://orcid.org/0000-0002-3433-0185 Pontifícia Universidade Católica de Goiás, Brasil E-mail: enzomugayar@gmail.com

Guilherme Pazinato Ritter ORCID: https://orcid.org/0000-0001-7898-8790 Pontifícia Universidade Católica de Goiás, Brasil E-mail: guilhermeritter2000@gmail.com

Gustavo Felipe Oliveira Barros ORCID: https://orcid.org/0000-0002-9806-9457 Pontifícia Universidade Católica de Goiás, Brasil E-mail: gustavofelipe2798@gmail.com

João Bosco Rocha Coimbra Neto ORCID: https://orcid.org/0000-0002-8487-560X Pontifícia Universidade Católica de Goiás, Brasil E-mail: netojoaobosco@hotmail.com

Antonio Márcio Teodoro Cordeiro Silva ORCID: https://orcid.org/0000-0003-0645-3599 Pontifícia Universidade Católica de Goiás, Brasil E-mail: marciocmed@gmail.com

Rogério José de Almeida ORCID: https://orcid.org/0000-0002-2150-6057 Pontifícia Universidade Católica de Goiás, Brasil E-mail: rogeriopucgo@gmail.com

\begin{abstract}
Resumo
O período da pandemia trouxe inúmeras mudanças sociais e acadêmicas de forma abrupta para a formação médica. $\mathrm{O}$ objetivo deste estudo foi avaliar o impacto que a pandemia trouxe para formação dos estudantes de medicina. Trata-se de um estudo transversal analítico com abordagem quantitativa. Dois questionários foram aplicados, sendo um com dados sociodemográficos, pessoais e acadêmicos e o outro foi a Escala Continuum de Saúde Mental versão reduzida (MHC-SF) para adultos. Foram incluídos na pesquisa 274 estudantes de medicina, dos quais $(63,5 \%)$ eram do sexo feminino e (36,5\%) do sexo masculino. A maioria dos participantes estava na faixa etária entre 21 a 25 anos $(58,4 \%)$. Piores escores da condição de saúde mental foram identificados no sexo feminino, nos estudantes do $1^{\circ}$ ao $4^{\circ}$ período, pelos que afirmaram não ter religião, que não realizaram atividades extracurriculares, não encontraram com os amigos, não praticaram exercícios físicos, que possuíam doença psiquiátrica diagnosticada, que tinham insônia, que não estavam satisfeitos com o rendimento acadêmico e que já pensaram em abandonar o curso de medicina. O presente estudo revelou diversos fatores associados a condição de saúde mental dos estudantes de medicina no período da pandemia de Covid19. Ademais, expõe-se os agravos emocionais, sociais e psicológicos implicados pelas medidas de distanciamento social e adoção de ensino a distância nos acadêmicos, sendo imprescindível a adoção de medidas para combater e prevenir o desenvolvimento dessa condição de saúde mental.
\end{abstract}

Palavras-chave: Covid-19; Educação médica; Estudantes de medicina; Saúde mental.

\begin{abstract}
The pandemic period brought numerous social and academic changes abruptly to medical education. The aim of this study was to assess the impact that the pandemic had on the education of medical students. This is an analytical crosssectional study with a quantitative approach. Two questionnaires were applied, one with sociodemographic, personal, and academic data and the other was the Continuum Mental Health Scale, reduced version (MHC-SF) for adults. The survey included 274 medical students, of whom $(63.5 \%)$ were female and $(36.5 \%)$ male. Most participants were aged between 21 and 25 years (58.4\%). Worst scores of mental health status were identified in females, in students from 1st to 4th period, by those who claimed to have no religion, who did not perform extracurricular activities, did not meet
\end{abstract}


friends, did not exercise, who had a diagnosed psychiatric illness, who had insomnia, who were not satisfied with their academic performance and who had already thought about dropping out of medical school. The present study revealed several factors associated with the mental health condition of medical students during the Covid-19 pandemic period. Furthermore, it exposes the emotional, social, and psychological damages implied by the measures of social distancing and the adoption of distance learning among students, being essential the adoption of measures to combat and prevent the development of this mental health condition.

Keywords: Covid-19; Medical education; Medical students; Mental health.

\section{Resumen}

El período de la pandemia trajo abruptamente numerosos cambios sociales y académicos a la educación médica. El objetivo de este estudio fue evaluar el impacto que tuvo la pandemia en la educación de los estudiantes de medicina. Se trata de un estudio analítico transversal con enfoque cuantitativo. Se aplicaron dos cuestionarios, uno con datos sociodemográficos, personales y académicos y el otro fue la Escala de Salud Mental Continuum, versión reducida (MHC-SF) para adultos. La encuesta incluyó a 274 estudiantes de medicina, de los cuales $(63,5 \%)$ eran mujeres y $(36,5 \%)$ hombres. La mayoría de los participantes tenían entre 21 y 25 años $(58,4 \%)$. Los peores puntajes de estado de salud mental fueron identificados en mujeres, en estudiantes del $1^{\circ}$ al $4^{\circ}$ período, por aquellos que decían no tener religión, que no realizaban actividades extracurriculares, no se reunían con amigos, no hacían ejercicio, que tenían una enfermedad psiquiátrica diagnosticada, que tenían insomnio, que no estaban satisfechos con su rendimiento académico y que ya habían pensado en abandonar la facultad de medicina. El presente estudio reveló varios factores asociados con la condición de salud mental de los estudiantes de medicina durante el período de la pandemia Covid-19. Además, expone los daños emocionales, sociales y psicológicos que implican las medidas de distanciamiento social y la adopción de la educación a distancia entre los estudiantes, siendo imprescindible la adopción de medidas para combatir y prevenir el desarrollo de esta condición de salud mental.

Palabras clave: Covid-19; Educación médica; Estudiantes de medicina; Salud mental.

\section{Introdução}

A atividade médica repassa uma ideia de uma profissão nobre que salva vidas, como uma escolha de doação de si mesmo para com o outro. O curso de medicina, em virtude de sua carga horária extensa e de período integral, além de atividades extracurriculares exigidas para uma formação ampla e completa, requer de seus discentes uma dedicação exímia e íntegra à faculdade. Ademais, a resistência física e mental que os estudantes devem desenvolver durante a sua formação visando o perfeccionismo e a autoexigência podem impactar de forma negativa a saúde mental (Santos et al., 2017).

A saúde mental é definida como o equilíbrio emocional entre suas necessidades e as exigências ou vivências externas. É a capacidade de administrar a própria vida e as suas emoções dentre as variadas situações, sem deixar de fazer o que é necessário (Zaidhaft, 2019). A saúde mental, social e emocional são interligadas e profundamente dependentes umas das outras. Portanto, perante a rotina sobrecarregada que os discentes do curso de medicina possuem, manter sua saúde íntegra torna-se algo extremamente difícil (Cortez et al., 2017).

A crescente produção científica acerca da saúde mental dos estudantes de medicina expõe uma realidade de preocupação em relação ao aumento de doenças que perpassam a sanidade mental desse grupo específico de pessoas em comparação com a população em geral. Os estudantes de medicina geralmente optam por estratégias psicológicas de negação, transgressão da culpa, isolamento social e o silêncio perante as adversidades - criando um ciclo que execra a sanidade mental desses acadêmicos e dificulta ainda mais a reversão desses quadros psíquicos (Conceição et al., 2019).

Se a condição geral da saúde mental do estudante de medicina já é fator de preocupação em tempos normais, durante o enfrentamento de uma pandemia torna-se ainda mais preocupante. A Coronavírus Disease 2019 (Covid-19) é um dos maiores desafios que a saúde pública mundial enfrenta atualmente. Tal doença foi identificada pela primeira vez em Wuhan, na província de Hubei, República Popular da China, em dezembro de 2019. A rápida disseminação doença em escala global fez com que a Organização Mundial da Saúde (OMS), em março de 2020, a considerasse uma pandemia (Maia \& Dias, 2020).

Com o objetivo de reduzir o curso ascendente da infecção pela doença, os governos adotam algumas medidas sociais, tais como: o isolamento de pessoas infectadas, fechamento de lugares públicos sujeitos à aglomeração, o distanciamento de 
pessoas consideradas grupo de risco para a doença, bem como a quarentena para toda a população e, em alguns casos mais rigorosos, o lockdown (Kissler et al., 2020).

Existem diversos fatores que podem contribuir para as manifestações de ansiedade e depressão em pessoas que estão vivenciando o isolamento social por conta da pandemia, ainda que ambos podem ser impulsionados por reações subsequentes ao estresse. É fato também que a falta de controle perante a essa situação nova, bem como o contexto cercado de impossibilidades e incertezas levam os indivíduos ao limite de sua sanidade mental (Pereira et al., 2020).

Logo, medidas com o propósito de reduzir implicações psicológicas, sociais e emocionais não podem ser desconsideradas nesse momento (Schmidit et al., 2020). Ainda nesse contexto, faz-se necessário citar a problemática envolvendo os estudantes de medicina que tiveram mudanças drásticas em suas vidas acadêmicas, passando de um cenário presencial para outro totalmente virtual. Aulas que antes eram dinâmicas, exigiam interação entre as pessoas, tinham um fluxo de ideias que eram trocadas, refutadas e esclarecidas, agora passaram para um ambiente monótono, digital e completamente novo. Contudo, as obrigações continuaram, a pressão para com o indivíduo aumentou e o bem-estar emocional, social e psicológico foi deixado de lado (Lima, 2020).

De fato, estudantes de medicina estão vulneráveis à manifestação de transtornos mentais, como estresse, depressão e ansiedade. O desenvolvimento desses transtornos pode interferir no bem-estar psicossocial dos estudantes, nas suas relações interpessoais e no seu desempenho acadêmico. Muitas vezes, os acadêmicos começas a manifestar cansaço excessivo e dificuldade de atenção e memória como sintomas iniciais. Posteriormente, começam a faltar aulas, não desenvolver mais suas atividades acadêmicas e, em alguns casos, acabam por abandonar o curso (Costa et al. 2020).

Nesse sentido, o foco da análise da problematização empreendida situa-se na avaliação da saúde mental de estudantes de medicina em meio à pandemia. Assim, o presente estudo teve por objetivo analisar como se encontra a condição de saúde mental dos estudantes de medicina perante o cenário de pandemia causado pela Covid-19.

\section{Metodologia}

Trata-se de um estudo transversal analítico com abordagem quantitativa. Este é um método de pesquisa observacional que tem como objetivo analisar dados coletados a partir de tempo e de uma população pré-definidos (Aragão, 2011).

A pesquisa foi realizada por meio de questionários aplicados aos estudantes de medicina de todo país. Não envolveu nenhuma universidade em particular, já que toda pesquisa se desenvolveu em formato digital por meio de redes sociais, como WhatsApp, E-mail e Facebook. O link contendo o Termo de Consentimento Livre e Esclarecido (TCLE) e os questionários foi encaminhado pelos pesquisadores a grupos que fazem parte e a colegas estudantes de medicina de várias partes do país. Foi solicitado aos colegas estudantes de medicina que replicassem esse link em suas redes sociais e e-mails constituindo uma amostragem por "bola de neve" (snow ball).

Para que a pesquisa fosse realizada ainda em um momento de pandemia com a utilização de aulas remotas nos cursos de medicina, optou-se que a coleta de dados fosse realizada entre os dias 01 e 11 de dezembro de 2020, ou seja, final de semestre em uma experiência totalmente nova com aulas remotas e em isolamento social.

Critérios de inclusão: discentes de medicina, com idade igual ou maior que 18 anos e que cursaram medicina durante a pandemia causada pela Covid-19. Critérios de exclusão: discentes que não estavam frequentando o curso e que não responderam a todas as questões dos questionários.

Para o desenvolvimento da pesquisa foram utilizados dois instrumentos de pesquisa. O primeiro foi um questionário sociodemográfico, pessoal e acadêmico criado pelos pesquisadores com variáveis de interesse para a pesquisa e que, pela literatura científica, se relacionam o fenômeno investigado. 
O segundo instrumento foi a Escala Continuum de Saúde Mental versão reduzida (MHC-SF) para adultos. Foi desenvolvido por Keyes (2002), adaptada e validada no Brasil por Machado e Bandeira (2015). Essa escala foi desenvolvida de acordo com vários modos de compreender a realidade perante ao bem-estar emocional, psicológico e social.

Ela está dividida em 14 itens de autorrelato: três itens para o bem-estar emocional (itens 1, 2 e 3 - escore varia entre 3 e 18), cinco itens para o bem-estar social (itens 4, 5, 6, 7 e 8 - escore varia entre 5 e 30) e seis itens para o bem-estar psicológico (itens $9,10,11,12,13$ e 14 - escore varia entre 6 e 36). Já o escore global é composto por todos os 14 itens com os escores variando entre 14 e 84. Menores escores indicam uma pior saúde mental dos estudantes (Machado \& Bandeira, 2015). versão reduzida da Escala MHC-SF tem obtido excelente consistência interna e validade para a população universitária, assim como sensibilidade e estabilidade a alterações temporais (Ferreira, 2015).

Com os dados coletados foi confeccionado um banco de dados utilizando o software IBM SPSS Statistics 18. Posteriormente, foi realizada estatística descritiva com o cálculo das frequências absoluta e relativa percentual, bem como do desvio padrão. Na sequência foi aplicado teste de normalidade (Kolmogorov-Smirnov) para distinguir as distribuições paramétricas e não-paramétricas, com o intuito de comparação dos resultados do questionário estratificado pelas variáveis sociodemográficas. Foram utilizados, para as distribuições paramétricas, os testes t de Student e ANOVA e para as distribuições não-paramétricas os testes Mann-Whitney e Kruskal-Wallis. Para todos os testes comparativos será assumido p-valor menor ou igual a 0,05 como significativo.

Antes de iniciar a coleta de dados, o presente trabalho foi encaminhado ao Comitê de Ética em Pesquisa (CEP) da Pontifícia Universidade Católica de Goiás (PUC Goiás), obtendo sua aprovação com o parecer n. 4.423 .451 em 26 de novembro de 2020 .

\section{Resultados}

Foram entrevistados 274 estudantes de medicina, cujo perfil sociodemográfico identificado foi do sexo feminino (63,5\%) e masculino (36,5\%), na faixa etária dos 21 aos 25 anos de idade (58,4\%), com um envolvimento religioso fraco (54,4\%) e que moravam com familiares $(75,9 \%)$ (Tabela 1$)$.

Tabela 1. Caracterização dos aspectos sociodemográficos dos 274 estudantes de medicina, Goiânia, Goiás, Brasil, 2020.

\begin{tabular}{|c|c|c|}
\hline Variável (N=274) & $n$ & $f(\%)$ \\
\hline \multicolumn{3}{|l|}{ Sexo } \\
\hline Feminino & 174 & 63,5 \\
\hline Masculino & 100 & 36,5 \\
\hline \multicolumn{3}{|l|}{ Idade } \\
\hline 18 a 20 anos & 94 & 34,3 \\
\hline 21 a 25 anos & 160 & 58,4 \\
\hline 26 a 30 anos & 16 & 5,8 \\
\hline Mais que 30 anos & 4 & 1,5 \\
\hline \multicolumn{3}{|l|}{ Período/Módulo } \\
\hline $1^{\circ}$ até $4^{\circ}$ & 123 & 44,9 \\
\hline $5^{\circ}$ até $8^{\circ}$ & 109 & 39,8 \\
\hline $9^{\circ}$ até $12^{\circ}$ & 42 & 15,3 \\
\hline \multicolumn{3}{|l|}{ Estado Civil } \\
\hline Solteiro(a) & 156 & 56,9 \\
\hline Solteiro(a)/Namorando & 112 & 40,9 \\
\hline Casado(a) & 6 & 2,2 \\
\hline \multicolumn{3}{|l|}{ Envolvimento Religioso } \\
\hline Nenhum & 28 & 10,2 \\
\hline
\end{tabular}




\begin{tabular}{lcc} 
Fraco & 149 & 54,4 \\
Forte & 63 & 23,0 \\
Não tenho religião & 34 & 12,4 \\
Com Quem Mora & & \\
Sozinho(a) & 49 & 17,9 \\
Amigos & 17 & 6,2 \\
Familiares & 208 & 75,9 \\
\hline
\end{tabular}

Fonte: Autores (2021).

Com relação aos aspectos pessoais e acadêmicos, identificou-se que os acadêmicos realizaram atividades extracurriculares $(81,8 \%)$, tiveram algum contato com amigos $(92,7 \%)$, praticaram atividade física de maneira regular $(74,1 \%)$, não eram portadores de doenças crônicas $(92,3 \%)$, não possuíam doença psiquiátrica $(76,3 \%)$ e nem faziam algum tipo de terapia $(66,1 \%)$. Há de se ressaltar também que houve demonstração de problemas com o sono $(75,3 \%)$, relataram ser etilistas $(73,4 \%)$, não estavam satisfeitos com o desempenho acadêmico (79,9\%), e a referiram que qualidade de vida piorou $(54,7 \%)$ (Tabela 2).

Tabela 2. Caracterização dos aspectos pessoais e acadêmicos dos 274 estudantes de medicina, Goiânia, Goiás, Brasil, 2020.

\begin{tabular}{|c|c|c|}
\hline Variável (N=274) & $n$ & $f(\%)$ \\
\hline \multicolumn{3}{|c|}{ Faz Atividade Extracurricular } \\
\hline Sim & 224 & 81,8 \\
\hline Não & 50 & 18,2 \\
\hline \multicolumn{3}{|c|}{ Encontrou com Amigos } \\
\hline Não encontrei & 20 & 7,3 \\
\hline Raramente & 110 & 40,1 \\
\hline Às vezes & 90 & 32,8 \\
\hline Frequentemente & 54 & 19,7 \\
\hline \multicolumn{3}{|c|}{ Praticou Atividade Física } \\
\hline Não pratiquei & 23 & 8,4 \\
\hline Raramente & 48 & 17,5 \\
\hline Às vezes & 72 & 26,3 \\
\hline Frequentemente & 131 & 47,8 \\
\hline \multicolumn{3}{|l|}{ Tem Doença Crônica } \\
\hline Sim & 21 & 7,7 \\
\hline Não & 253 & 92,3 \\
\hline \multicolumn{3}{|c|}{ Tem Doença Psiquiátrica } \\
\hline Sim & 65 & 23,7 \\
\hline Não & 209 & 76,3 \\
\hline \multicolumn{3}{|c|}{ Fez Terapia Psiquiátrica } \\
\hline Sim & 93 & 33,9 \\
\hline Não & 181 & 66,1 \\
\hline \multicolumn{3}{|l|}{ Insônia } \\
\hline Sim & 68 & 24,8 \\
\hline Não & 206 & 75,2 \\
\hline \multicolumn{3}{|l|}{ Horas de Sono/Noite } \\
\hline Menos de 7 horas & 116 & 42,3 \\
\hline Entre 7 e 9 horas & 153 & 55,8 \\
\hline Mais de 9 horas & 5 & 1,8 \\
\hline \multicolumn{3}{|l|}{ Etilista } \\
\hline Sim & 201 & 73,4 \\
\hline Não & 73 & 26,6 \\
\hline
\end{tabular}




\section{Tabagista}

Sim

Não

Satisfeito com Rendimento Acadêmico

Sim

Não

Pensou Abandonar o Curso

Sim

86

188

31,4

Não

Realizar Outras Atividades

Sim

Não

Na comparação dos aspectos sociodemográficos com os níveis da condição de saúde mental, identificou-se no domínio do bem-estar emocional um pior escore em relação ao sexo feminino ( $p<0,0001)$. No domínio do bem-estar social o pior escore foi representado pelo sexo feminino $(\mathrm{p}<0,0001)$, pelos estudantes do $1^{\circ}$ ao $4^{\circ}$ período $(\mathrm{p}=0,0210)$ e por aqueles que afirmaram não ter religião $(\mathrm{p}=0,0066)$. No domínio do bem-estar psicológico o menor escore novamente foi representado pelo sexo feminino ( $p<0,0001)$. Por fim, no escore global de saúde mental, menor escore foi identificado sexo feminino ( $<<0,0001)($ Tabela 3).

Tabela 3. Comparação dos aspectos sociodemográficos com os níveis da condição de saúde mental dos 274 estudantes de medicina, Goiânia, Goiás, Brasil, 2020.

\begin{tabular}{|c|c|c|c|c|c|c|c|c|c|c|c|c|}
\hline \multirow{2}{*}{ Variável (N=274) } & \multicolumn{2}{|c|}{ Emocional } & \multirow{2}{*}{$p$-valor } & \multicolumn{2}{|c|}{ Social } & \multirow{2}{*}{$p$-valor } & \multicolumn{2}{|c|}{ Psicológico } & \multirow{2}{*}{$p$-valor } & \multicolumn{2}{|c|}{ Global } & \multirow{2}{*}{$p$-valor } \\
\hline & Média & $D P$ & & Média & $D P$ & & Média & $D P$ & & Média & $D P$ & \\
\hline \multicolumn{13}{|l|}{ Sexo } \\
\hline Feminino & 12,2 & 3,1 & & 13,8 & 5,2 & & 21,4 & 6,3 & & 47,5 & 13,0 & \\
\hline Masculino & 14,1 & 2,7 & $<0,0001$ & 17,5 & 5,8 & $<0,0001$ & 26,0 & 6,1 & $<0,0001$ & 57,6 & 13,3 & $<0,0001$ \\
\hline \multicolumn{13}{|l|}{ Idade } \\
\hline 18 a 20 anos & 13,1 & 3,2 & & 15,0 & 5,7 & & 22,9 & 7,1 & & 50,9 & 14,6 & \\
\hline 21 a 25 anos & 12,8 & 3,0 & & 15,2 & 5,8 & & 23,1 & 6,5 & & 51,2 & 14,0 & \\
\hline Mais de 25 anos & 13,2 & 3,3 & 0,8258 & 15,1 & 5,1 & 0,9527 & 24,3 & 4,8 & 0,6823 & 52,6 & 11,0 & 0,8925 \\
\hline \multicolumn{13}{|l|}{ Período/Módulo } \\
\hline $1^{\circ}$ até $4^{\circ}$ & 12,8 & 3,2 & & 14,3 & 5,9 & & 22,8 & 7,2 & & 49,9 & 14,9 & \\
\hline $5^{\circ}$ até $8^{\circ}$ & 13,3 & 2,7 & & 16,1 & 5,1 & & 23,3 & 5,9 & & 52,7 & 12,4 & \\
\hline $9^{\circ}$ até $12^{\circ}$ & 12,4 & 3,8 & $0,5876^{*}$ & 15,1 & 6,0 & $0,0210 *$ & 23,4 & 6,5 & 0,7748 & 50,9 & 15,2 & 0,3286 \\
\hline \multicolumn{13}{|l|}{ Estado Civil } \\
\hline Solteiro(a) & 13,1 & 3,0 & & 15,3 & 5,4 & & 23,0 & 6,7 & & 51,3 & 13,7 & \\
\hline Solteiro(a)/Namorando & 12,8 & 3,3 & & 15,0 & 6,1 & & 23,2 & 6,7 & & 51,0 & 14,7 & \\
\hline Casado(a) & 12,0 & 3,6 & 0,6261 & 13,5 & 5,6 & 0,7421 & 25,0 & 2,8 & 0,7679 & 50,5 & 10,5 & 0,9770 \\
\hline \multicolumn{13}{|l|}{ Envolvimento Religioso } \\
\hline Nenhum & 12,6 & 3,1 & & 13,3 & 5,7 & & 22,4 & 6,7 & & 48,3 & 13,9 & \\
\hline Fraco & 12,9 & 3,0 & & 15,4 & 5,6 & & 23,0 & 6,7 & & 51,3 & 14,2 & \\
\hline Forte & 13,4 & 3,3 & & 16,5 & 5,2 & & 24,0 & 6,0 & & 54,0 & 13,2 & \\
\hline Não tenho religião & 12,3 & 3,2 & 0,3010 & 12,9 & 5,8 & 0,0066 & 22,7 & 7,0 & 0,6496 & 47,9 & 14,2 & 0,1310 \\
\hline \multicolumn{13}{|l|}{ Com Quem Mora } \\
\hline Sozinho(a) & 12,5 & 3,5 & & 14,7 & 5,8 & & 22,4 & 5,9 & & 49,6 & 13,4 & \\
\hline Amigos & 12,8 & 3,0 & & 16,2 & 5,7 & & 23,5 & 6,1 & & 52,5 & 13,9 & \\
\hline Familiares & 13,0 & 3,0 & 0,5744 & 15,2 & 5,7 & 0,6450 & 23,2 & 6,8 & 0,7114 & 51,4 & 14,2 & 0,6687 \\
\hline
\end{tabular}

Testes utilizados: teste t de Student e ANOVA; $\left(^{*}\right)$ teste de Mann-Whitney e Kruskal-Wallis. Nível de significância de 5\%. Fonte: Autores (2021). 
Na comparação dos aspectos pessoais e acadêmicos com os níveis da condição de saúde mental, identificou-se no domínio do bem-estar emocional que piores escores foram encontrados nas seguintes variáveis: não realizar atividades extracurriculares $(\mathrm{p}=0,0008)$, não encontrar com os amigos $(\mathrm{p}=0,0017)$, não praticar exercícios físicos $(\mathrm{p}=0,0015)$, ter doença psiquiátrica ( $\mathrm{p}=0,0006)$, fazer terapia psiquiátrica/psicológica $(\mathrm{p}=0,0090)$, ter insônia $(\mathrm{p}=0,0001)$, dormir mais de 9 horas de sono por noite $(\mathrm{p}=0,0196)$, não estar satisfeito com o rendimento acadêmico $(\mathrm{p}=0,0345)$, ter pensado em abandonar o curso $(\mathrm{p}=0,0008)$, não realizar outras atividades durante o curso $(\mathrm{p}=0,0005)$ e nos que afirmaram que sua qualidade de vida havia piorado na pandemia $(\mathrm{p}<0,0001)$ (Tabela 4).

Sobre o domínio do bem-estar social, identificaram-se piores escores nas seguintes variáveis: não encontrar os amigos frequentemente $(\mathrm{p}=0,0018)$, não praticar atividades físicas $(\mathrm{p}=0,0006)$, ter doença psiquiátrica $(\mathrm{p}<0,0001)$, realizar terapia psiquiátrica/psicológica $(\mathrm{p}=0,0112)$, ter insônia $(\mathrm{p}=0,0386)$, ter pensado em abandonar o curso $(\mathrm{p}=0,0001)$ e nos que afirmaram que sua qualidade de vida havia piorado na pandemia ( $\mathrm{p}<0,0001)$ (Tabela 4).

Já no domínio do bem-estar psicológico, encontraram-se piores escores nas variáveis: não realizar atividades extracurriculares $(\mathrm{p}=0,0259)$, não encontrar os amigos $(\mathrm{p}=0,0095)$, não praticar atividades física $(\mathrm{p}<0,0001)$, ter doença psiquiátrica $(\mathrm{p}<0,0001)$, realizar terapia psiquiátrica/psicológica $(\mathrm{p}=0,0453)$, ter insônia $(\mathrm{p}=0,0077)$, não estar satisfeito com o rendimento acadêmico ( $\mathrm{p}=0,0001)$, ter pensado em abandonar o curso $(\mathrm{p}<0,0001)$, não realizar outras atividades além do curso de medicina $(\mathrm{p}<0,0001)$ e nos que afirmaram que sua qualidade de vida havia piorado na pandemia $(\mathrm{p}<0,0001)$ (Tabela 4).

Por fim, no escore global da escala, obteve-se menores escores nas variáveis: não realizar atividades extracurriculares $(\mathrm{p}=0,0082)$, não encontrar os amigos $(\mathrm{p}=0,0013)$, não praticar atividades físicas $(\mathrm{p}<0,0001)$, ter doença psiquiátrica $(\mathrm{p}<0,0001)$, realizar terapia psiquiátrica/psicológica $(\mathrm{p}=0,0108)$, ter insônia $(\mathrm{p}=0,0035)$, não estar satisfeito com o rendimento acadêmico $(\mathrm{p}=0,0084)$, ter pensado em abandonar o curso $(\mathrm{p}<0,0001)$, não realizar outras atividades durante o curso $(\mathrm{p}=0,0001)$ e nos que afirmaram que sua qualidade de vida havia piorado na pandemia ( $\mathrm{p}<0,0001)$ (Tabela 4).

Tabela 4. Comparação dos aspectos pessoais e acadêmicos com os níveis da condição de saúde mental dos 274 estudantes de medicina, Goiânia, Goiás, Brasil, 2020.

\begin{tabular}{|c|c|c|c|c|c|c|c|c|c|c|c|c|}
\hline \multirow{2}{*}{ Variável (N=274) } & \multicolumn{2}{|c|}{ Emocional } & \multirow{2}{*}{$p$-valor } & \multicolumn{2}{|c|}{ Social } & \multirow{2}{*}{$p$-valor } & \multicolumn{2}{|c|}{ Psicológico } & \multirow{2}{*}{$p$-valor } & \multicolumn{2}{|c|}{ Global } & \multirow{2}{*}{$p$-valor } \\
\hline & Média & $D P$ & & Média & $D P$ & & Média & $D P$ & & Média & $D P$ & \\
\hline \multicolumn{13}{|c|}{ Fez Atividade Extracurricular } \\
\hline Sim & 13,3 & 2,9 & & 15,5 & 5,5 & & 23,5 & 6,3 & & 52,2 & 13,3 & \\
\hline Não & 11,4 & 3,6 & $0,0008 *$ & 13,6 & 6,3 & $\mathbf{0 , 0 3 0 9}$ & 21,5 & 7,8 & $\mathbf{0 , 0 2 5 9}$ & 46,4 & 16,2 & $\mathbf{0 , 0 0 8 2}$ \\
\hline \multicolumn{13}{|c|}{ Encontrou com Amigos } \\
\hline Não encontrei & 10,4 & 3,3 & & 10,8 & 4,4 & & 18,5 & 5,1 & & 39,7 & 10,9 & \\
\hline Raramente & 12,9 & 3,3 & & 14,9 & 5,5 & & 23,0 & 7,0 & & 50,8 & 14,3 & \\
\hline Às vezes & 13,4 & 2,7 & & 15,7 & 5,6 & & 23,8 & 5,7 & & 52,9 & 12,7 & \\
\hline Frequentemente & 13,2 & 2,9 & $\mathbf{0 , 0 0 1 7}$ & 16,3 & 5,8 & 0,0018 & 23,8 & 7,0 & 0,0095 & 53,3 & 14,6 & 0,0013 \\
\hline \multicolumn{13}{|c|}{ Praticou Atividade Física } \\
\hline Não pratiquei & 10,5 & 3,5 & & 10,7 & 3,6 & & 17,1 & 5,1 & & 38,3 & 10,4 & \\
\hline Raramente & 12,8 & 3,6 & & 15,4 & 6,1 & & 22,5 & 7,1 & & 50,7 & 15,3 & \\
\hline Às vezes & 12,7 & 3,0 & & 14,7 & 5,4 & & 22,8 & 6,4 & & 50,2 & 13,6 & \\
\hline Frequentemente & 13,5 & 2,6 & $0,0015^{*}$ & 16,1 & 5,6 & 0,0006 & 24,6 & 6,1 & $<0,0001$ & 54,2 & 13,0 & $<0,0001$ \\
\hline \multicolumn{13}{|c|}{ Tem Doença Crônica } \\
\hline Sim & 12,1 & 3,6 & & 14,0 & 5,5 & & 22,3 & 5,4 & & 48,4 & 13,1 & \\
\hline Não & 13,0 & 3,1 & 0,2263 & 15,2 & 5,7 & 0,3232 & 23,2 & 6,7 & 0,5776 & 51,4 & 14,1 & 0,3518 \\
\hline \multicolumn{13}{|c|}{ Tem Doença Psiquiátrica } \\
\hline Sim & 11,8 & 3,4 & & 12,6 & 5,2 & & 20,0 & 5,8 & & 44,4 & 12,9 & \\
\hline Não & 13,3 & 2,9 & 0,0006 & 15,9 & 5,6 & $<0,0001$ & 24,1 & 6,5 & $<0,0001$ & 53,3 & 13,7 & $<0,0001$ \\
\hline \multicolumn{13}{|c|}{ Fez Terapia Psiquiátrica } \\
\hline Sim & 12,2 & 3,3 & & 13,9 & 5,2 & & 22,0 & 6,2 & & 48,2 & 13,5 & \\
\hline Não & 13,3 & 2,9 & 0,0090 & 15,8 & 5,8 & $\mathbf{0 , 0 1 1 2}$ & 23,7 & 6,7 & $\mathbf{0 , 0 4 5 3}$ & 52,7 & 14,0 & 0,0108 \\
\hline
\end{tabular}




\begin{tabular}{|c|c|c|c|c|c|c|c|c|c|c|c|c|}
\hline \multicolumn{13}{|l|}{ Insônia } \\
\hline Sim & 11,5 & 3,5 & & 14,1 & 5,8 & & 21,3 & 6,5 & & 46,9 & 14,5 & \\
\hline Não & 13,4 & 2,8 & $0,0001 *$ & 15,5 & 5,6 & $\mathbf{0 , 0 3 8 6}$ & 23,7 & 6,5 & $\mathbf{0 , 0 0 7 7}$ & 52,6 & 13,5 & 0,0035 \\
\hline \multicolumn{13}{|l|}{ Horas de Sono/Noite } \\
\hline Menos de 7 horas & 12,6 & 3,4 & & 15,5 & 6,0 & & 22,6 & 7,1 & & 50,7 & 15,3 & \\
\hline Entre 7 e 9 horas & 13,3 & 2,8 & & 15,0 & 5,5 & & 23,6 & 6,2 & & 51,9 & 12,9 & \\
\hline Mais de 9 horas & 10,2 & 3,4 & $\mathbf{0 , 0 1 9 6}$ & 11,2 & 3,8 & 0,2180 & 20,0 & 6,4 & 0,2845 & 41,4 & 13,1 & 0,2288 \\
\hline \multicolumn{13}{|l|}{ Etilista } \\
\hline Sim & 13,0 & 3,0 & & 15,4 & 5,9 & & 23,3 & 6,6 & & 51,6 & 14,1 & \\
\hline Não & 12,9 & 3,4 & 0,8196 & 14,5 & 5,1 & 0,2633 & 22,5 & 6,5 & 0,3887 & 49,9 & 13,6 & 0,3630 \\
\hline \multicolumn{13}{|l|}{ Tabagista } \\
\hline Sim & 12,6 & 2,7 & & 15,9 & 6,2 & & 23,5 & 8,1 & & 52,0 & 16,0 & \\
\hline Não & 13,0 & 3,1 & 0,5671 & 15,1 & 5,6 & 0,5305 & 23,1 & 6,5 & 0,7819 & 51,1 & 13,9 & 0,7969 \\
\hline \multicolumn{13}{|c|}{ Satisfeito com Rendimento } \\
\hline Sim & 13,6 & 3,2 & & 15,9 & 6,2 & & 26,1 & 6,0 & & 55,6 & 14,1 & \\
\hline Não & 12,8 & 3,1 & 0,0345 & 14,9 & 5,5 & 0,2799 & 22,3 & 6,5 & $\mathbf{0 , 0 0 0 1}$ & 50,1 & 13,8 & 0,0084 \\
\hline \multicolumn{13}{|c|}{ Pensou Abandonar o Curso } \\
\hline Sim & 12,0 & 3,0 & & 13,2 & 5,0 & & 20,7 & 6,1 & & 45,8 & 12,8 & \\
\hline Não & 13,4 & 3,0 & 0,0008 & 16,0 & 5,7 & 0,0001 & 24,2 & 6,5 & $<0,0001$ & 53,6 & 13,9 & $<0,0001$ \\
\hline \multicolumn{13}{|c|}{ Realizar Outras Atividades } \\
\hline Sim & 13,3 & 2,9 & & 15,4 & 5,5 & & 23,9 & 6,2 & & 52,6 & 13,2 & \\
\hline Não & 11,7 & 3,6 & 0,0005 & 14,1 & 6,1 & 0,1038 & 20,1 & 7,1 & $<0,0001$ & 45,8 & 15,5 & 0,0001 \\
\hline \multicolumn{13}{|c|}{ Qualidade de Vida Piorou } \\
\hline Sim & 12,0 & 3,0 & & 13,4 & 5,1 & & 21,0 & 5,9 & & 46,4 & 12,5 & \\
\hline Não & 14,1 & 2,8 & $<0,0001$ & 17,2 & 5,7 & $<0,0001$ & 25,7 & 6,5 & $<0,0001$ & 57,0 & 13,5 & $<0,0001$ \\
\hline
\end{tabular}

Testes utilizados: teste t de Student e ANOVA; (*) teste de Mann-Whitney e Kruskal-Wallis. Nível de significância de 5\%. Fonte: Autores (2021).

\section{Discussão}

O estudo evidenciou um perfil de estudantes de medicina em que as mulheres representaram a maioria da amostra, bem como um predomínio de acadêmicos mais jovens, entre 17 e 25 anos, seguindo e confirmando as tendências do último Censo de Educação Superior (INEP, 2019). A crescente quantidade de mulheres médicas configura um processo denominado "feminização da medicina". Esse fenômeno desperta uma nova foram de atuação da medicina que leva em questão a luta histórica das mulheres em relação a opressão de gêneros e a segurança na igualdade das leis (Rodrigues, 2020).

Quanto em relação ao sexo, pode-se concluir que no domínio do bem-estar emocional as mulheres atingiram piores escores. Revalidando esse dado, um estudo realizado no curso de medicina de uma universidade do Sudoeste da Bahia concluiu que $32,2 \%$ dos alunos entrevistados apresentavam algum transtorno mental comum, desses 70,3\% eram do sexo feminino (Santos et al., 2017). As mulheres possuem uma maior predisposição ao estresse e, consequentemente, a desenvolverem desordens mentais. Possíveis causas desse maior risco estão relacionadas com os inúmeros papéis que as mulheres desenvolvem na sociedade, com as mudanças hormonais mais significativas vivenciadas por elas e com a importância de um apoio social para elas (Machado et al., 2020).

A inserção dos jovens estudantes brasileiros no meio acadêmico da medicina traz uma infinidade de novas experiências. Com tamanha cobrança desde os primeiros períodos, muitos discentes acabam afetados com a rotina agitada e rígida exigida pelo curso, haja vista que estão vulneráveis às novas mudanças e imaturos com as novas vivências. Desse modo, muitos acabam por não reconhecerem seus próprios adoecimentos, principalmente aqueles relacionados a questões psicossociais, pois entendem que isso não passa de uma "adaptação" à nova rotina, o que pode propiciar um adoecimento precoce. Ademais, esse adoecimento repercute ao longo de todo o curso, visto que o não reconhecimento e o não tratamento dessas condições piora, cada vez mais, o desempenho acadêmico, a vivência social e a condição de saúde mental do estudante de medicina (Conceição et al., 2019).

Quando comparado o envolvimento religioso e os escores nos aspectos emocional, social e psicológico, identificou-se que os alunos que não possuíam nenhuma prática religiosa ou que não possuíam religião apresentaram piores escores. Isso, pois, 
identifica-se, por mais tênue que seja, uma conexão entre a ciência e a religiosidade. Ambas, quando correlacionadas, podem contribuir positivamente em um certo processo de ressignificação dos estudantes, em desacordo com o sofrimento ou adoecimento mental dos alunos (Vieira, Barros \& Firmino, 2020).

Atividades extracurriculares são essenciais para o desenvolvimento socioprofissional dos estudantes. No presente estudo, notou-se que quem não desenvolve atividades extracurriculares atingiram escores emocionais, sociais e psicológicos estatisticamente menores quando comparados aos alunos que desenvolviam. Essas práticas permitem os alunos a desenvolverem algumas atividades que a graduação em si pode não proporcionar, despertando o sentimento de os alunos estarem mais próximos de como desenvolverão sua profissão. Assim, a falta de execução dessas atividades extracurriculares que as medidas de enfrentamento à pandemia proporcionaram também desmotiva alguns alunos a se dedicarem nos estudos, até mesmo, a permanecerem no curso, tendo em vista que se sentem cada vez mais distantes de suas atividades acadêmicas e de suas práticas profissionais (Machado et al., 2020).

Ademais, para a execução dessas atividades, o estudante de medicina deve ter uma dedicação integral para com o curso, o que limita seu tempo livre e acaba por afetar funções fisiológicas, psicológicas e cognitivas. Assim os acadêmicos ficam inseridos em uma conjuntura em que se descuidam das próprias necessidades e não praticam atividade física. A prática regular da atividade física está relacionada a uma boa saúde mental, a melhora da capacidade de superação e interação social, fatores cruciais para um período de pandemia, ainda mais quando inseridos no meio dos acadêmicos de medicina. Cabe ressaltar também que o sedentarismo pode piorar algumas condições do sono, como a insônia, piorando, consequentemente, o nível de fadiga do discente (Machado et al., 2020; Vaz et al., 2020).

O estado de bem-estar mental, psicológico e social é de suma importância como condição de confronto para com as situações adversas que os estudantes enfrentam durante o curso. É fato inconteste que o auxílio psicológico e/ou terapia psiquiátrica têm papel fundamental para a manutenção de uma boa saúde mental e no auxílio e formulação de estratégias de contorno aos contratempos e obstáculos que surgem diariamente no curso de medicina, como o isolamento, noites mal dormidas, estresse cotidiano, ansiedade e angústia para com a rotina do dia a dia (Machado et al., 2020).

Um estudo realizado com 116 internos de curso de medicina da cidade de Goiânia/GO demonstrou que existe uma íntima relação entre a sonolência excessiva diurna e o desempenho acadêmico. Esse fato é influenciado diretamente pelos estudantes que apresentam insônia ou utilizam de alguma substância para estimular o sono. Corroborando a isso, observa-se que os estudantes que apresentaram insônia estavam mais insatisfeitos com o seu rendimento acadêmico quando comparados aos que conseguem dormir bem. A redução desse rendimento é uma consequência direta dos prejuízos advindos de uma noite mal dormida, posto que essa causa um decaimento das funções cognitivas, como a atenção, a concentração, a autoestima e, até mesmo, o humor (Vaz et al., 2020).

Além disso, uma das consequências notadas perante esse cenário pandêmico é a dificuldade que o estudante lida em manter seu rendimento acadêmico de forma satisfatória. A mudança do método presencial para o método remoto influenciou no desempenho dos alunos, posto que o último exige novos métodos de estudo e uma disciplina diferente da antiga, mesmo o curso exigindo as mesmas demandas de antes. Não são todos os alunos que conseguem adaptar-se de forma rápida e eficiente perante as mudanças no formato de ensino. Esse fato instiga alguns estudantes a pensarem em abandonar o curso (Medeiros, 2020).

A pandemia de Covid-19 trouxe várias medidas de distanciamento entre as pessoas como forma de conter a propagação do vírus. Entretanto, o isolamento social já é uma realidade sórdida entre aqueles que moram longe dos entes queridos, pouco se encontram com amigos (ou as vezes nem isso) e vivenciam todos os dias a angústia das incertezas para com essa doença. Os impactos mentais associados à quarentena são agravados quando a pessoa possui alguma doença psiquiátrica e/ou necessita de terapia, devido a maior fragilidade mental do indivíduo e maior dificuldade de lidar com as situações cotidianas. Outrossim, 
problemas com o sono, atividade física e ansiedade tornaram-se ainda mais frequentes, mesmo em indivíduos antes tidos como pessoas sadias (Brooks, 2020).

O presente estudo levanta questões relevantes sobre a educação médica e sobre a prática da medicina. Sendo assim, é de suma importância entender as causas e as consequências dos problemas psicológicos que os estudantes desenvolvem ao longo de sua formação. Contudo, as instituições responsáveis pelo aprendizado médico devem extrapolar a identificação e o entendimento de tais problemas e passar a intervir nesses, de tal forma que se promova o bem-estar nos acadêmicos. Promover tal bem-estar e nutri-lo durante a formação dos estudantes se faz algo essencial para o desenvolvimento de habilidades que os tornem bons profissionais e para a construção de uma boa base da resiliência que irá acompanhá-los ao longo de toda sua carreira. Essa ação, a longo prazo, não irá beneficiar apenas os estudantes, mas também o ambiente onde estão inseridos, os pacientes que serão atendidos e, assim, a população em geral (Conceição et al., 2019).

\section{Conclusão}

De fato, a pandemia causada pelo coronavírus afetou a rotina de grande parte de cidadãos pelo mundo, inclusive dos estudantes de medicina. Essas mudanças de rotina, a incerteza causada pela pandemia e o isolamento social, entre outras causas, afetaram diretamente a saúde mental desses estudantes.

Os indícios de uma saúde mental debilitada que foram estudados demonstraram alguns pontos significantes dentro da população que foi estudada. Foi possível concluir que existem fatores sociodemográficos de risco para o desenvolvimento de uma saúde mental ruim nos estudantes me medicina durante a pandemia da Covid-19, sendo eles: ser do sexo feminino, ter 18 a 20 anos, estar entre o $5^{\circ}$ e o $8^{\circ}$ período e não ter nenhum envolvimento religioso. Além desses fatos, também existem os aspectos pessoais e acadêmicos que favorecem o desenvolvimento de desajustes em relação à saúde mental, como: não encontrar os amigos, não praticar atividades físicas, ter alguma doença crônica, ter alguma doença psiquiátrica ou realizar terapia psiquiátrica, por exemplo.

Diante dos resultados obtidos, evidencia-se a importância de se desenvolver mais discussões que abordem a saúde mental dos estudantes de medicina, com o objetivo de fornecer um suporte psicossocial mais adequado para os estudantes. Além disso, esses resultados podem ser úteis para o desenvolvimento de algumas ações que as instituições acadêmicas adotem visando prevenir a presença de sintomas relacionados à saúde mental e abordagens mais efetivas para a população que foi estudada em um contexto de pandemia.

\section{Referências}

Aragão, J. (2011). Introdução aos estudos quantitativos utilizados em pesquisas científicas. Revista Práxis, 3(6), 59-62.

Barros, M. B. A. et al. (2020). Relato de tristeza/depressão, nervosismo/ansiedade e problemas de sono na população adulta brasileira durante a pandemia de COVID-19. Epidemiologia e Serviços de Saúde, 29(4), e2020427.

Brooks, S. K. et al. (2020). The psychological impact of quarantine and how to reduce it: rapid review of the evidence. The Lancet, 395(10227) 912-920.

Conceição, L. S. et al. (2019). Saúde mental dos estudantes de medicina brasileiros: uma revisão sistemática da literatura. Revista da Avaliação da Educação Superior, 24(3), 785-802.

Cortez, E. A. et al. (2017). Promoção à saúde mental dos estudantes universitários. Revista Pró-UniverSUS, 8(1), $48-54$.

Costa, D. S. et al. (2020). Sintomas de depressão, ansiedade e estresse em estudantes de medicina e estratégias institucionais de enfrentamento. Revista Brasileira de Educação Médica, 44(1), e040.

Ferreira, I. G. et al. (2016). Extracurricular activities and medical formation: diversity and curricular flexibility. Interdisciplinary Journal of Health Education, $1(2), 114-124$.

Ferreira, C. (2015). Caracterização da saúde mental numa população jovem de concelho da Póvoa de Varzim: da psicopatologia ao bem-estar. 2015. Dissertação (Mestrado em Psicologia) - Instituto de Psicologia, Universidade do Porto, Porto. 117. 
Research, Society and Development, v. 10, n. 16, e596101623933, 2021

(CC BY 4.0) | ISSN 2525-3409 | DOI: http://dx.doi.org/10.33448/rsd-v10i16.23933

Instituto Nacional De Estudos e Pesquisas Educacionais Anísio Teixeira (INEP). (2019). Censo de Educação Superior. Ministério da Educação, 2019. Disponível em: https://download.inep.gov.br/educacao_superior/censo_superior/documentos /2020/Notas_Estatisticas_Censo_da_Educacao_Superior_2019.pdf >. Acesso em: 14 set. 2021.

Kissler, S. M. et al. (2020). Projecting the transmission dynamics of SARS-COV-2 through the postpandemic period. Science, 368(6493), 860-868.

Lima, R. C. (2020). Distanciamento e isolamento social pela covid-19 no brasil: impactos na saúde mental. Revista de Saúde Coletiva, 30(2) 1-10.

Machado, J. N. et al. (2020). Fatores associados aos níveis de estresse percebido em estudantes internos de um curso de medicina. Revista Brasileira Militar de Ciências, 06(16).

Machado, W. L., \& Bandeira, D. R. (2015). Positive mental health scale: validation of the mental health continuum. Programa de Pós-Graduação em Psicologia da Universidade de São Francisco, 20(2), 259-274.

Maia, B. R., \& Dias, P. C. (2020). Anxiety, depression, and stress in university students: the impact of COVID-19. Estudos de Psicologia, 37 , e200067.

Medeiros, E. A. S. (2020). Desafios para o enfrentamento da pandemia da COVID-19 em hospitais universitários. Revista Paulista de Pediatria, 38.

Pereira, M. D. et al. (2020). A Pandemia de COVID-19, o isolamento social, consequências na saúde mental e estratégias de enfrentamento: uma revisão integrativa. Research Society and Development, 9(7), 1-35.

Rodrigues, O. M. (2020). Bioética e gênero: o processo de feminização da medicina no estado de São Paulo e a conformação de coletivos feministas. FCMUNICAMP

Santos, L. S. et al. (2017). Qualidade de vida e transtornos mentais comuns em estudantes de medicina. Cogitare Enfermagem, $22(4)$, e52126.

Schmidt, B. et al. (2020). Impacts on mental health and psychological interventions related to the new coronavirus pandemic (COVID-19). Estudos de Psicologia, $37, \mathrm{e} 200063$.

Vaz, et al. (2020). Fatores Associados aos Níveis de Fadiga e Sonolência Excessiva Diurna em Estudantes do Internato de um Curso de Medicina. Revista Brasileira de Educação Médica, 44(1).

Vieira, E. S., Barros, M. M. M., \& Firmino, R. G. (2020). Religiosidade e saúde mental: visão de equipe multiprofissional de centro de atenção psicossocial. Cadernos Brasileiros de Saúde Mental, 12(33), 16-40.

Zaidhaft, S. (2019). A saúde mental dos estudantes de medicina: reminiscências e conjecturas de um mestre-escola. Revista de Medicina, 98(2), 86-98. 